\title{
DESENVOLVIMENTO DO SCHISTOSOMA MANSONI DAS LINHAGENS DE BELO HORIZONTE (MG), E DE SÃO JOSE DOS CAMPOS (SP). EM MUS MUSCULUS
}

\begin{tabular}{l|l|} 
RSPU-B/179 \\
\hline
\end{tabular}

Magalhães, L. A. \& Carvalho, J. F. de - Desenvolvimento do Schistosoma mansoni das linhagens de Belo Horizonte (MG), e de São José dos Campos (SP), em Mus musculus. Rev. Saúde públ., S. Paulo, 7: 285-7, 1973.

Resumo: Foram analisados os dados referentes ao desenvolvimento de cercárias de $\mathrm{S}$. mansoni das linhagens de Belo Horizonte (MG) e de São José dos Campos (SP), Brasil. Concluiu-se que após a penetração das cercárias pelo tegumento dos camundongos, näo houve diferença significativa quanto ao numero de vermes adultos que se desenvolveram, pertencentes às duas cepas estudadas.

UNITERMos: Schistosoma mansoni*; Esquistossomose *

\section{I $\mathbf{N}$ T $R$ O O D U C $\mathbf{A}$ O}

Em trabalho anterior verificamos diferença significativa entre o número de cercárias de $S$. mansoni, provenientes de Minas Gerais e de São Paulo, que penetravam pela cauda de Mus musculus (Magalhães \& CarvalHo 1). Determinamos que, em média, $93 \%$ das cercárias da linhagem de Belo Horizonte penetravam pela cauda dos camundongos e que, nas mesmas condições, $79,1 \%$ das cercárias provenientes de São José dos Campos, SP, infectaram os roedores.

Na presente nota, estudamos o número médio de esquistossomas que conseguem completar seu desenvolvimento nos animais assinalados.

\section{MATERIAL E METODOS}

Infectamos dois lotes de 10 camundongos, pesando aproximadamente de 14 a $16 \mathrm{~g}$, com 100 cercárias por roedor das linhagens de $S$. mansoni de Belo Horizonte (MG), e de São José dos Campos (SP), respectivamente, pelo método utilizado para a verificação da viabilidade de penetração das cercárias (MAgalhães 2).

Sessenta dias após o inóculo, foram perfundidos os camundongos sobreviventes à infecção, e contados os esquistossomas encontrados na Veia Porta e seus ramos mesentéricos e hepáticos.

* Do Instituto de Biologia da Universidade Estadual de Campinas. - Caixa Postal 1170 Campinas, S.P. - Brasil.

* Do Instituto de Ciências Matemáticas de São Carlos. Universidade de São Paulo São Carlos, S.P. - Brasil. 
MAGALHAES, L. A. \& CARVALHo, J. F. de - Desenvolvimento do Schistosoma mansoni das linhagens de Belo Horizonte (MG), e de São José dos Campos (SP), em Mus musculus. Rev. Saúde públ., S. Paulo, 7:285-7, 1973.

R E S U L T A D O $\mathbf{S}$

Obtivemos os resultados constantes das Tabelas 1 e 2 .

T A $\quad$ B $\quad$ E $\quad$ L A $A$

Dados referentes à penetração e desenvolvimento de cercárias de $S$. mansoni das cepas de Belo Horizonte (BH) e São José dos Campos (SJ) expostas à infecção pela cauda de Mus musculus.

\begin{tabular}{|c|c|c|c|c|}
\hline Espec. & \multirow{2}{*}{ Nāo penetraram } & \multicolumn{2}{|c|}{ Penetraram } & \multirow{2}{*}{ TOTAL } \\
\hline Cepa & & Desenvolvidas & Não desenvolvidas & \\
\hline BH & 30 & 165 & 805 & 1000 \\
\hline SJ & 210 & 123 & 667 & 1000 \\
\hline
\end{tabular}

T A B E L A 2

Dados referentes ao desenvolvimento de 1000 cercárias das linhagens de Belo Horizonte (BH) e São José dos Campos (SJ) expostas à infecção pela cauda de 10 Mus musculus.

\begin{tabular}{l|c|c|c}
\cline { 3 - 3 } & $\begin{array}{c}\text { Desen- } \\
\text { volvidas }\end{array}$ & $\begin{array}{c}\text { Náo de- } \\
\text { senvol- } \\
\text { vidas }\end{array}$ & TOTAL \\
\hline BH & 165 & 805 & 970 \\
SJ & 123 & 667 & 790 \\
\hline
\end{tabular}

DISCUSAAO E CONCLUSAO

Utilizamos duas amostras independentes, uma para a cepa de $\mathrm{BH}$ e outra para a cepa de SJ, obtendo-se os resultados constantes da Tabela 1.

Desejamos saber se os dois grupos diferem em alguma característica (por exemplo, se há alguma diferença significativa entre as frequiências relativas). Aplicamos, então, teste baseado na distribuição $X^{2}$ (Siegel ${ }^{3}$ ).
Encontramos,

$$
\mathrm{X}_{2}^{2}=152,2
$$

valor que é significativo a $5 \%$.

Logo, concluimos que há diferença en. tre as cepas estudadas.

Prosseguindo a análise, estudamos do mesmo modo, quanto ao desenvolvimento, apenas as cercárias que penetraram no hospedeiro definitivo (Tabela 2).

Obtivemos:

$$
\mathrm{X}^{2}{ }_{1}=0,559
$$

valor não significativo a $\mathbf{5 \%}$.

Logo não há evidência da diferença entre as cepas, relativamente ao desenvolvimento de cercárias que penetraram.

Finalmente, podemos também concluir que a diferença encontrada na Tabela 1 deve-se somente à diferença na capacidade de penetração. 
MAGALHAES, L. A. \& CARVALHO, J. F. de - Desenvolvimento do Schistosoma mansoni das linhagens de Belo Horizonte (MG), e de São José dos Campos (SP), em Mus musculus. Rev. Saúde públ., S. Paulo, 7:285-7, 1973.

RSPU-B/179

Magalhĩes, L. A. \& Carvalho, J. F. de - [Development of Schistosoma mansoni strains from Belo Horizonte, MG and S. José dos Campos, SP (Brazil) in Mus musculus.] Rev. Saúde públ., S. Paulo, 7: 285-7, 1973.

Summary: Experimental data of the developing of $\mathbf{S}$. mansoni cercariae of two strains (Belo Horizonte, MG and S. José dos Campos, SP), penetrating through the tails of Mus musculus, were analysed. The data led to conclude that there is no numerical difference, in the two strains, as for development from cercariae to adult worm.

UNITERMS: Schistosoma mansoni *; Schistosomiasis *.

\section{REFERENCIAS BIBLIOGRAFICAS}

1. magalhaES, L. A. \& CARVAlHo, J. F. Determinação do número de cercárias provenientes de cepas diferentes de Schistosoma mansoni que conseguem penetrar, sob determinadas condiçóes de laboratório, em Mus musculus. Rev. Soc. bras. Med. trop., 5:249-51, 1968.
2. MAGALHAES, I. A. - Técnica para avaliação da viabilidade de penetração de cercárias de Schistosoma mansoni em Mus musculus. Hospital, Rio de Janeiro, 75:1663-6, 1969.

3. SIEGEL, S. - Nonparametric statistics for the behavioral sciences. New York, Mc Graw Hill, 1956. p. 104.

Recebido para publicaçāo em 15-6-1973

Aprovado para publicaçăo em 10-7-1973 